\title{
Using the Dispersal Assembly Hypothesis to predict local species richness from the relative abundance of species in the regional species pool
}

\author{
D. R. Schoolmaster Jr
}

\author{
Department of Biology, University of Michigan, Ann Arbor, Michigan 48109, USA. \\ Email:dschoolm@umich.edu
}

Keywords: Diversity, Model, Prediction.

\begin{abstract}
In recent years, many authors have attempted to determine whether local processes (such as competitive exclusion) result in local species richness below that of a local community assembled only by dispersal from a larger regional species pool. To do this they hypothesize that no local processes are significant and that local communities are assembled by dispersal only (Dispersal Assembly Hypothesis or DAH). Some authors have presumed that a prediction of this hypothesis is that, if many regions of similar ecological type (e.g., grassland, temperate deciduous forest, etc.) are compared then local richness will be the same fixed proportion of regional richness, across all regions. To compare this putative prediction with observed data, they plot local richness on the vertical axis vs. regional richness on the horizontal axis and discover how well a straight line fits: if it fits well then they accept the hypothesis and they take the slope as an estimate of the fixed proportion; if it fits poorly (usually by curving down for larger regional pools), then they reject the hypothesis and they presume that local processes are significant. In the present paper, I hypothesize DAH, and for each of two different species relative abundance distributions and predict, by simulation, the probability distribution of species richness in a local community, for a full spectrum of local community sample sizes. The plot of the expected values of these predicted distributions vs. regional richness (for a constant local sample size) and vs. increasing local sample size (for constant regional richness) is not a straight line in either case, but always curves downward. Thus, a straight line (proportional sampling) is never a prediction of DAH, so that looking for straight lines in data plots is irrelevant to testing DAH. Finally, I describe how to compare these predictions to observed data to test whether local processes significantly limit local species richness.
\end{abstract}

Abbreviation: DAH - Dispersal Assembly Hypothesis(es)

\section{Introduction}

One of the main goals of community ecology is to attempt to explain patterns of species diversity. Suggested explanations of observed patterns of richness differ in the spatial scale at which the mechanisms responsible for creating and maintaining species diversity are supposed to work: some suggest that the processes important in determining species diversity operate on the local level, and others suggest that only regional level processes are important (Ricklefs 1987, Cornell and Lawton 1992, Cornell 1993). Theories based on local processes hypothesize that interspecific interactions, such as competition and predation limit which species can occur (Hutchinson 1959, Mac Arthur 1972, Tilman 1982). Communities structured by these factors have been called 'Niche assembly communities' by Hubbell (1997). Theories based on regional processes hypothesize that dispersal, and not biotic interactions, determine the number of species that co-occur in a local area. These theories predict that the numbers of species available to colonize each local area from the surrounding region determine the number of species in that local area (Lawton 1982, Cornell 1985, Caley and Schluter 1997). Communities structured by these factors have been called 'Dispersal assembly' communities by Hubbell (1997). Local and regional processes are both undoubtedly present in all communities, the challenge to ecologists is to create a methodology to discover which set of processes are most important to determining species richness in a given community.

Terborgh and Faaborg (1980) and Cornell (1993) have tested whether local processes are important by comparing the local richness in a series of 'roughly uniform' habitats that are subject to colonization from regional species pools of different sizes. In this way, they compared local species richness among standardized samples taken in comparable habitats. Cornell (1993) and Cornell and Karlson (1997) suggest making this comparison by plotting local richness as the dependent variable 
and regional richness as the independent variable. They have suggested that the shape of the resulting plot can indicate the scale at which the mechanisms responsible for maintaining species diversity occur, as shown in Figure 1. They argue that, if local interactions are absent, the number of species in local areas will be a constant proportion of the number of species in the regional species pool. If this were the case, then the plot would appear as a straight line. They refer to such a straight line as 'Proportional sampling'. An extreme case of this would be if all the species in a region were present in each local area. The line on Figure 1 marked 'Boundary' (slope $=1$ ) would represent this case. They claim that if local richness remains constant for all regional richness values above an intermediate threshold (a straight line does not fit well), then strong local interactions limit the number of species that can exist in the local area beyond the limitation of dispersal (Cornell 1993). This situation is indicated on Figure 1, and has been referred to as 'Saturation.'

A number of studies employing this method have been published for a wide array of organisms including: free-living and parasitic insects, helminth parasites, corals, fish, lizards, birds and plants (Lawton 1999, Srivastava 1999). A recent review of these studies concludes that the proportional sampling phenomenon is common (Cornell and Karlson 1997). However, Huston (1999), Caley and Schluter (1997) and Srivastava (1999) have pointed out that the authors of these studies have used a variety of concepts of regional and local scale and have measured these pools in different ways, and Srvistava (1999) has pointed out that the shapes of the curves have been analyzed in many different ways, including simple linear regression, curvilinear, and log-log regressions,

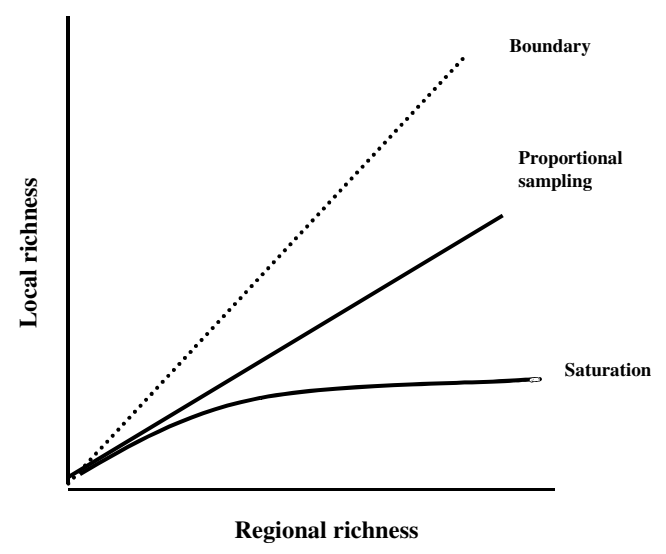

Figure 1. Predictions of Terborg and Faaborg (1980) and Cornell $(1985,1993)$ for potential shapes of local richness vs. regional richness plots. with the choice of test sometimes affecting the conclusion.

In addition to these problems with implementing this method, the present work examines three problems with the logical basis of this method. Specifically, 1) the hypothesis that local richness is determined by proportional dispersal from the regional pool (i.e., there are no local forces that limit local species richness) does not predict 'proportional sampling'. 2) Regions are compared without considering the relative abundance of species in the regional species pool. 3) It incorrectly assumes that all local areas are the same size, i.e., measured with the same sampling effort, or that there will be no effect of different local area sizes on the predictions.

One way to test if local factors limit species richness is to hypothesize that local species richness is limited only by dispersal. Below, this hypothesis will be called the Dispersal Assembly Hypothesis (DAH). To test if observed data are consistent with $\mathrm{DAH}$, it is necessary to state the hypothesis in terms sufficiently specific that quantitative predictions can be deduced and compared with observed data. One of several ways to hypothesize a specific colonization process would encompass the idea that species more abundant in the regional species pool are more likely to colonize the local area than species less abundant in the regional pool. To state the dispersal assembly hypothesis in this way: species richness in a local area is solely the result of which species happens to colonize from the regional pool. The probability that any organism sampled from the regional pool is a particular species is proportional to its relative abundance in the regional pool, and is independent of what other species are sampled. Similar ideas have been incorporated into the colonization functions of metapopulation models (Hanski and Gilpin 1997).

\section{Methods}

The species richness of local areas measured by a sample of a given size was simulated by randomly sampling that number of individuals from the regional species pool. As hypothesized, the species identity of each individual was selected with the probability equal to its fraction of the total number of individuals in the regional pool. To estimate the predicted probability that a local area sample would have a particular richness, one thousand local areas were simulated. Such a predicted probability distribution was estimated for each of several combinations of local area size and regional pool relative abundance. In figures 4 through 7, the mean and fifth and ninety-fifth percentile of the probability distributions are reported. 


\section{Results}

Critique \#1. In general, 'Proportional sampling' is not predicted by the $D A H$.

To see if the DAH predicts that local species richness will be a constant proportion of the regional species pool, I simulated the DAH to predict probability distributions for local species richness for a number of regional pools that vary in species richness. I chose regional pool sizes and sampling efforts that are typical of published studies employing the local vs. regional graphical method.

The DAH is a hypothesis that asserts that no local processes reduce richness below that predicted by dispersal proportional to regional abundance. Ecological factors that have shaped relative abundance at the regional level are not in question here. So, I chose to examine two distinct distributions of regional relative abundance to determine the role regional abundance might play in the predictions of the DAH.

The two distributions of regional species abundances I am reporting here are, 1) all species equally abundant in the regional pool (Fig. 2), 2) species abundances log-normally distributed in the regional pool. If the species abundance has a log-normal distribution, then a few species are very abundant and most species are rare (Fig. 3). The results of these simulations are shown in Figures 4 and 5.

If the regional pool has a small number of equally abundant species and the number of individuals counted in the local area is large, the predicted species richness is nearly always equal to the richness of the regional pool, i.e., most of the species present in the regional pool appear in the local area. In this case, local species richness is a constant proportion $(=1)$ of the regional richness. As the richness of the regional species pool gets larger relative to

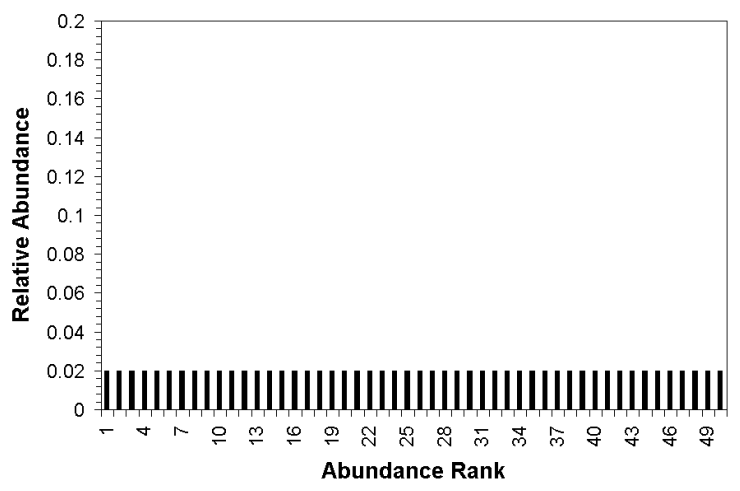

Figure 2. Uniform distribution of species relative abundances in a hypothetical regional pool containing 50 species.

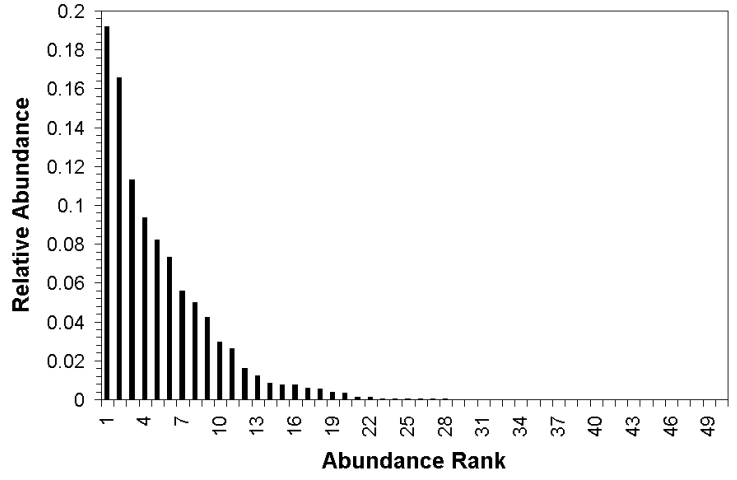

Figure 3. Log-normal distribution of species relative abundances in a hypothetical regional pool containing 50 species.

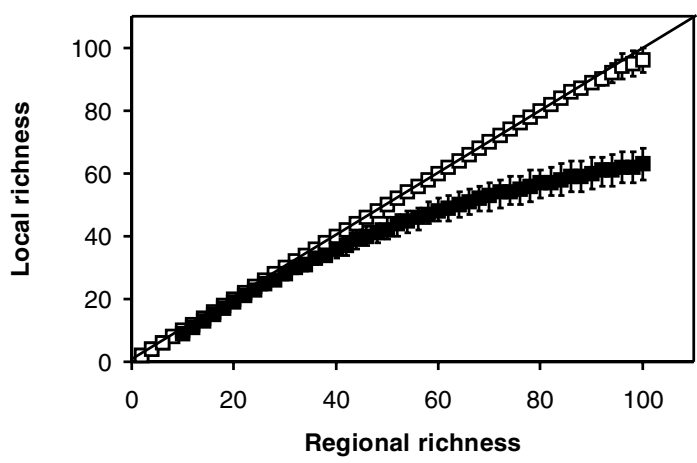

Figure 4. DAH predictions of local species richness where regional species abundances are uniformly distributed and the local area size equals 100 (filled squares) and 500 (open squares).

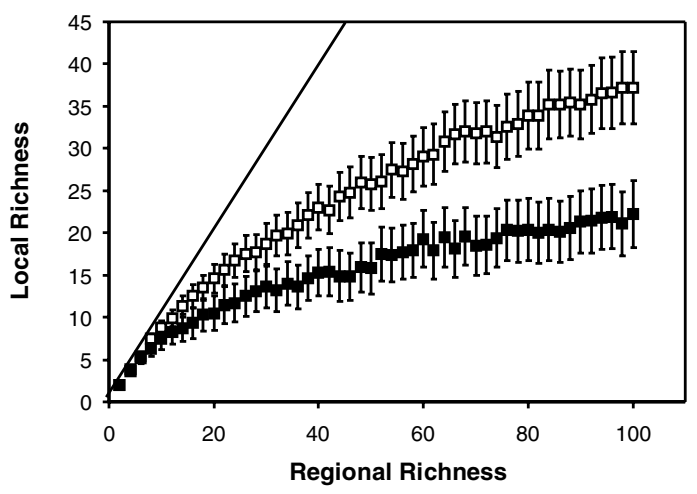

Figure 5. DAH predictions of local species richness where regional species abundances are log-normally distributed and the local area size equals 100 (filled squares) and 500 (open squares). 
the number of individuals counted in the local area, predicted species richness is dis-proportionally smaller. Thus, the curve that describes the relationship between predicted local species richness and the richness of the regional species pool bends down. This also occurs if relative abundances in the regional pool are log-normally distributed, provided that regional richness is large relative to local area size.

Critique \#2: Regions are compared without considering the distribution of species relative abundance in the regional species pool.

Studies that compare the local with regional richness among regions often do so without considering the distribution of species relative abundance in the different regional pools. To test how the distribution of species relative abundance in the regional pool can affect the predictions of the DAH, I compared its predicted probability distribution of local species richness using the two different distributions of species relative abundance in the regional pool (uniform and log-normal). Predictions were derived by sampling 100 and 500 individuals from each distribution of regional species relative abundance for a range of regional species pool richness from two to one hundred species (Fig. 6).

There is no difference in local species richness predicted by the two distributions of regional species relative abundances if the regional species richness is low (2-10 species). However, if the regional species richness is higher $(>10)$, then predicted local richness is greater when species are uniformly distributed in the regional species pool, than when they are log-normally distributed. Local area size (sampling effort) does not affect these results qualitatively. These predictions of the DAH show that the distribution of species relative abundance in the regional

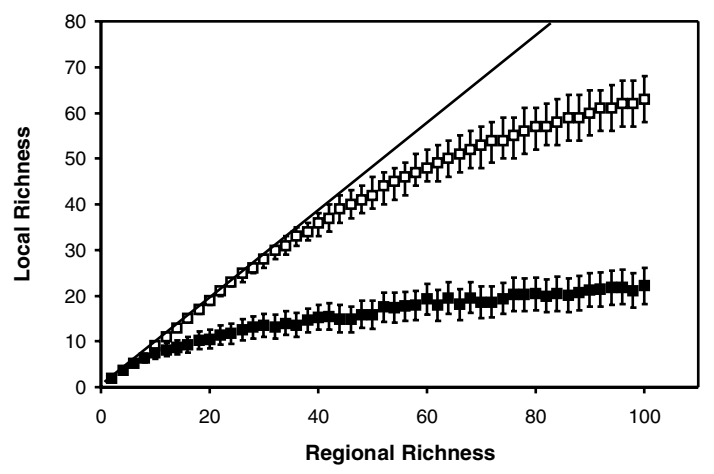

Figure 6. DAH predictions of local species richness where local area size equals 100 individuals and regional species abundances are uniformly distributed (open squares) and log-normally distributed (filled squares).

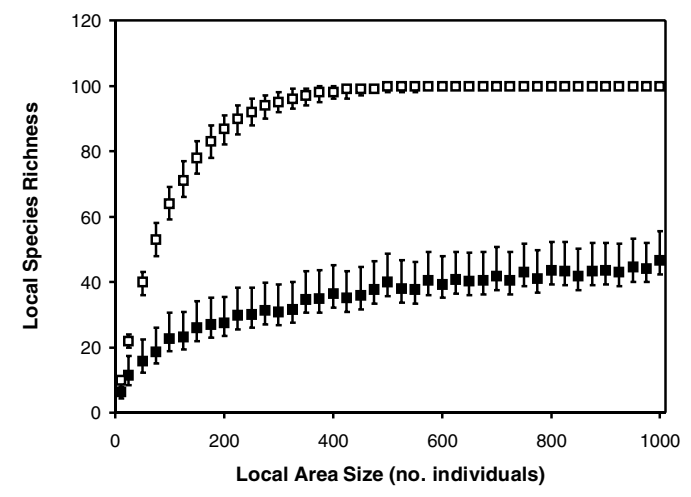

Figure 7. DAH predictions of local species richness if regional pool richness equals 100 species and relative abundances are uniformly distributed (open squares) or log-normally distributed (filled squares).

pool participate in making predictions of local species richness. In general, one cannot use the DAH to compare local species richness among regions without considering the underlying distributions of species relative abundance in the regional species pools.

\section{Critique \#3: Does local area size participate in the predictions of the DAH?}

Studies that compare local with regional species richness do so without considering how many individuals should be counted in each local area. To test how the numbers of individuals in the local area can affect predictions of the DAH, I compared predicted probability distributions of local species richness among local areas with different numbers of individuals. Figure 7 shows local richnesses predicted by the DAH with different local area sizes plotted against the number of individuals counted in the corresponding local area. Local species richness predicted by the DAH increases with a decreasing slope as the size of the local area increases. The distribution of relative abundances in the local area participates in DAH predictions by affecting the maximum predicted local species richness.

When comparing observed data to predictions of $\mathrm{DAH}$, one must be aware that the spatial scale at which the effects of local processes are observable is unknown. The following example demonstrates how local heterogeneity may affect conclusions as to the effect of local processes on local species richness. The solid and dashed curves of Figure 8 represent the richness of local area vs. the size of the local area in two areas that differ with respect to local heterogeneity. Area 1 (represented by the solid line) is more locally homogeneous so, local species 
richness increases quickly, but levels off quickly. Area 2 (represented by the dashed line) is more locally heterogeneous, so local species richness increases slowly with increasing area size before eventually leveling off. In locally homogeneous area 1 , low sampling effort would result in the conclusion that local species richness is consistent with the DAH. However, increasing sampling effort in area 1 would reveal a species richness significantly lower than predicted by DAH. In the locally heterogeneous area 2, relatively low sampling effort detects a local species richness lower than predicted by the DAH, but a larger sampling effort detects a local species richness consistent with the DAH. Thus, local area size (sampling effort) not only participates in DAH predictions but also participates in the observations that are to be compared to DAH.

\section{Practical considerations for testing DAH.}

To test DAH with field data, first measure the species richness and relative abundances of the regional pool, then choose a number of practical local area sizes. For each local area size, simulate the probability distributions of local area richness. Finally, observe the richness of the local areas, and compare them with the predicted species richness.

The regional pool is linked to the local area through dispersal. To quantify the species richness and relative abundance of the regional pool, one must discover, among the species that could potentially grow in the local area, which are actually getting there. The proportions of propagules for each of these species, among all that arrive to the local area, represent that species' relative abun-

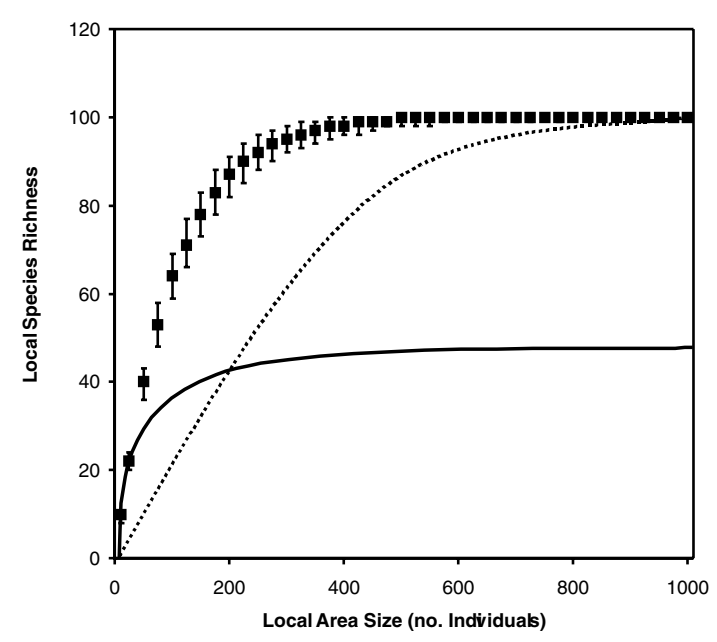

Figure 8. DAH predictions of local species richness (boxes) with hypothetical curves representing observed richness in a homogenous local area (solid line) and a heterogeneous local area (dashed line). dance in the regional species pool. When considering plants, seeds establishing from the seed bank, if any, must also be taken into account.

It may prove difficult or time consuming to make these observations, so that plausible estimates by less direct means become more justified. In any case, to use DAH to make a prediction, species membership and abundance in the pool of species that could colonize a local area need to be estimated. Once this has been done, information from a single region can be used to generate predictions of DAH. This eliminates the need to compare across regions, and assume that the same ecological forces are important in similar regions in different parts of the world. This method also allows predictions of the DAH to be tested for a number of different local area sizes, which is useful since the spatial scale at which limitation by local forces will occur is rarely known a priori.

\section{Discussion}

Terborgh and Faaborg (1980) and Cornell and Karlson (1997) suggested that the plot of local vs. regional richness measured from several different regions should be a straight line if local processes do not set a limit on species richness. Here, predictions from the DAH (local processes absent), clearly show that local richness is not a constant proportion of regional richness except in trivial cases.

Although Caley and Schluter (1997) have found that saturating curves result from sampling a regional pool with log-normally distributed species abundances, this result has not received much attention from Srivastava (1999) or Lawton (1999) who reviewed studies employing local-regional plots. Only by considering the sampling regime used and the relative abundances of species in the regional pools can the results of such studies be accurately assessed. In addition, collecting data from many distinct regions may result in a poor estimate of the regional relative abundance of species used to make predictions from the DAH to test for local richness limiting factors. The relative abundances of species in a single regional species pool can predict a probability distribution of richness for a given local area size with which data can be compared. This is an important point because it greatly reduces the effort needed to test a given community for the presence of local richness limiting factors, and eliminates the need to assume that different regions are the same, or even similar.

Recently, Caley and Schluter (1997), Srivastava (1999) and Huston (1999) have critically examined the assumptions and methods to compare local with regional 
richness to infer local processes. Such examination helps to discover the influence of regional species pools on the species richness of local communities, which will help us understand the processes that are important in maintaining species diversity. For this reason, it is necessary to devise a methodology that can clearly separate the influences of processes acting at each spatial scale. The approach I have described here provides a means to predict local richness from regional richness. This will enable us to determine more accurately whether we need to evoke local processes to explain low levels of local richness.

Acknowledgements: I would like to thank G.F. Estabrook for critical discussions on this topic. I am also grateful to G.F. Estabrook, C. Wetmore, M. Kummel, H. Possingham, and an anonymous reviewer for their help in preparing this manuscript.

\section{References}

Caley, M.J. and D. Schluter. 1997. The relationship between local and regional diversity. Ecology 78: 70-80.

Cornell, H.V. 1985. Species assemblages of cynipid gall wasps are not saturated. Amer. Nat. 126: 565-569.

Cornell, H.V. 1993. Unsaturated patterns in species assemblages: the role of regional processes in setting local species richness. In: R. E. Ricklefs and D. Schluter (eds.), Species Diversity in Ecological Communities. University of Chicago Press, Chicago, Illinois, USA, pp. 243-252

Cornell, H.V. and R.H. Karlson. 1997. Local and regional processes as controls of species richness. In: D. Tilman. and P. Kareiva (eds.). Spatial Ecology. The Role of Space in Population Dynamics and Interspecific Interactions. Princeton Univ. Press, Princeton, NJ, pp. 250-268.
Cornell, H.V. and J.H. Lawton. 1992. Species interactions, local and regional processes, and limits to the richness of ecological communities: a theoretical perspective. J. Animal Ecol. 61: 1-12.

Hanski, I. and M.E. Gilpin (eds.). 1997. Metapopulation Biology: Ecology, Genetics and Evolution. Academic Press, San Diego, CA.

Hubbell, S. 1997. A unified theory of biogeography and relative species abundance and its application to tropical rain forests and coral reefs. Coral Reefs 16. Supp1.: S9-S21.

Huston, M.A. 1999. Local processes and regional patterns: appropriate scales for understanding variation in the diversity of plants and animals. Oikos 86: 393-401.

Hutchinson, G.E. 1959. Homage to Santa Rosalia, or why are there so many kinds of animals? Amer. Nat. 93: 145-159.

Lawton, J.H. 1982. Vacant niches and unsaturated communities: a comparison of braken herbivores at sites on two continents. $J$. Animal Ecol. 51: 573-595.

Lawton, J.H. 1999. Are there general laws in ecology? Oikos 84: 177-192.

MacArthur, R. H. 1972. Geographical Ecology. Princeton University Press, Princeton, NJ.

Ricklefs, R.E. 1987. Community diversity: relative roles of local and regional processes. Science 235: 167-171.

Srivastava, D.S. 1999. Using local-regional richness plots to test for species saturation: pitfalls and potentials. J. Animal Ecol. 68: 1-16.

Terborgh, J.W. and J. Faaborg. 1980. Saturation of bird communities in the West Indies. Amer. Nat. 116: 178-195.

Tilman, D. 1982. Resource Competition and Community Structure. Princeton University Press, Princeton, NJ. 